\title{
A DISCRETE WAVELET ANALYSIS OF FREAK WAVES IN THE OCEAN
}

\author{
EN-BING LIN AND PAUL C. LIU
}

Received 25 June 2003 and in revised form 7 June 2004

\begin{abstract}
A freak wave is a wave of very considerable height, ahead of which there is a deep trough. A case study examines some basic properties developed by performing wavelet analysis on a freak wave. We demonstrate several applications of wavelets and discrete and continuous wavelet transforms on the study of a freak wave. A modeling setting for freak waves will also be mentioned.
\end{abstract}

\section{Introduction}

In the past few years, wavelet methods have been applied in coastal and ocean current data analysis $[2,5,6]$. These new tools have better performance than the traditional Fourier techniques. They localize the information in the time-frequency space and are capable of trading one type of resolution for the other, which renders them suitable for the analysis of nonstationary signals. It was first introduced in $[5,6]$ that the wavelet transform is a powerful tool for coastal and ocean engineering studies. An analysis of applying continuous wavelet transform spectrum analysis to the result of laboratory measurement of landslide-generated impulse waves was presented in [2]. In fact, the measured results are understandably unsteady, nonlinear, and nonstationary, hence the application of timelocalized wavelet transform analysis is shown to be a suitable as well as a useful approach. The analysis of correlating the time-frequency wavelet spectrum configurations in connection with the ambient parameters that drive the impulse wave process, with respect to time and space, leads to interesting and stimulating insights not previously known. In [7], an analysis of a set of available freak wave measurements gathered from several periods of continuous wave recordings made in the Sea of Japan during 1986-1990 by the Ship Research Institute of Japan was presented. The analysis provides an ideal opportunity to catch a glimpse of the incidence of freak waves. The results show that a well-defined freak wave can be readily identified from the wavelet spectrum, where strong energy density in the spectrum is instantly surged and seemingly carried over to the high-frequency components at the instant the freak wave occurs. Thus for a given freak wave, there appears a clear corresponding signature shown in the time-frequency wavelet spectrum. 
In fact, there is a considerable interest in understanding the occurrence of freak waves. There are a number of reasons why freak wave phenomena may occur. Often, extreme wave events can be explained by the presence of ocean currents or bottom topography that may cause wave energy to focus on a small area because of refraction, reflection, and wave trapping [3]. In this paper, we study wavelet analysis for a given time series, namely, we examine various wavelet tools on a given signal. We present several different aspects of wavelet analysis, for example, wavelet decomposition, discrete and continuous wavelet transforms, denoising, and compression, for a given signal. A case study examines some interesting properties developed by performing wavelet analysis in greater detail. We present a demonstration of the application of wavelets and wavelet transforms on waves. Here we use Daubechies wavelet (db3) in most of our applications unless otherwise specified in the context.

\section{Wavelet transform}

2.1. 1D continuous wavelet transforms. First, we review the standard continuous wavelet transforms. The continuous wavelet transform of a function $f(x) \in L^{2}(\mathbb{R})$ with respect to $\psi(x) \in L^{2}(\mathbb{R})$ is defined by

$$
(W f)(a, b)=\int_{-\infty}^{\infty} f(x) \psi^{a, b}(x) d x,
$$

where $a, b \in \mathbb{R}, a \neq 0$, and $\psi^{a, b}(x)$ is obtained from the function $\psi(x)$ by translating and dilating:

$$
\psi^{a, b}(x)=|a|^{-1 / 2} \psi\left(\frac{x-b}{a}\right)
$$

This transform is useful when one wants to recognize or extract features of the function $f(x)$ from the transform domain.

2.2. 1D discrete wavelet transforms (DWTs). Suppose $\phi(x)$ and $\psi(x)$ are the scaling function and the corresponding wavelet, respectively, with finite support $[0, l]$, where $l$ is a positive number. It is well known that $\phi(x)$ and $\psi(x)$ satisfy the following dilation equation:

$$
\begin{aligned}
& \phi(x)=\sqrt{2} \sum_{s=0}^{l} h_{s} \phi(2 x-s), \\
& \psi(x)=\sqrt{2} \sum_{s=0}^{l} g_{s} \phi(2 x-s),
\end{aligned}
$$

where the $h_{s}{ }^{\prime} s$ and $g_{s}$ 's are constants called lowpass and highpass filter coefficients, respectively [8]. 
We will use the following standard notations:

$$
\begin{aligned}
& \phi_{j, k}=2^{j / 2} \phi\left(2^{j}-k\right), \\
& \psi_{j, k}=2^{j / 2} \phi\left(2^{j}-k\right) .
\end{aligned}
$$

Consider the subspace $V_{j}$ of $L^{2}$ defined by

$$
V_{j}=\operatorname{Span}\left\{\phi_{j, k}, k \in \mathbb{Z}\right\}
$$

and the subspace $W_{j}$ of $L^{2}$ defined by

$$
W_{j}=\operatorname{Span}\left\{\psi_{j, k}, k \in \mathbb{Z}\right\}
$$

where the subspaces $V_{j}{ }^{\prime} s,-\infty<j<\infty$, form a multiresolution of $L^{2}$ with the subspace $W_{j}$ being the difference between $V_{j}$ and $V_{j-1}$. In fact, the $L^{2}$ space has an orthonormal decomposition as

$$
L^{2}=V_{j} \bigoplus \sum_{k=j}^{-\infty} W_{k}
$$

The projection of an $L^{2}$ function $f(x)$ onto the subspace $V_{j}$ is defined by

$$
f_{j}(x)=\sum_{k} \alpha_{j, k} \phi_{j, k}(x)
$$

where

$$
\alpha_{j, k}=\int f(x) \phi_{j, k}(x) d x
$$

Similarly, we can project $f(x)$ onto $W_{j}$ by

$$
w_{j}(x)=\sum_{k} \beta_{j, k} \phi_{j, k}(x)
$$

where

$$
\beta_{j, k}=\int f(x) \psi_{j, k}(x) d x
$$

Therefore, the function $f(x)$ can be decomposed by

$$
f(x)=f_{j}(x)+\sum_{i=j}^{-\infty} w_{i}(x) .
$$

The projection $f_{j}(x)$ is called the linear approximation of the function $f(x)$ in the subspace $V_{j}$. 
From (2.3)-(2.6), the projection coefficients $\alpha_{j, k}$ and $\beta_{j, k}$ of $f(x)$ in the subspaces $V_{j}$ and $W_{j}$ can be easily computed by the so-called fast wavelet transform:

$$
\alpha_{j, i}=\sum_{s=0}^{l} h_{s} \alpha_{j+1,2 i+s}, \quad \beta_{j, i}=\sum_{s=0}^{l} g_{s} \alpha_{j+1,2 i+s} .
$$

Unlike in the continuous case where the wavelet transform is applied to the $L^{2}$ function $f(x)$, in the discrete case, we start by considering a set of discrete numbers which are the low-frequency coefficients of the $L^{2}$ function $f(x)$ at a fine-level subspace $V_{j+1}$.

In practice, DWTs are very useful. It is a tool that cuts up data, functions, or operators into different frequency components, and then studies each component with a resolution matched to its scale.

Assume that $\left\{h_{k}, k \in \mathbb{Z}\right\}$ and $\left\{g_{k}, k \in \mathbb{Z}\right\}$ are lowpass and highpass filter coefficients, respectively, which satisfy (2.3) and (2.4); then for a discrete signal $\left\{a_{k}, k \in \mathbb{Z}\right\}$, the lowfrequency part $\left\{c_{k}, k \in \mathbb{Z}\right\}$ and the high-frequency part $\left\{d_{k}, k \in \mathbb{Z}\right\}$ of the DWT of $\left\{a_{k}\right\}$ are

$$
c_{k}=\sum_{l} h_{l-2 k} a_{l}, \quad d_{k}=\sum_{l} g_{l-2 k} a_{l}
$$

and the inverse DWT will give back $\left\{a_{k}\right\}$ from the knowledge of $\left\{c_{k}\right\}$ and $\left\{d_{k}\right\}$ :

$$
a_{k}=\sum_{l}\left(\tilde{h}_{k-2 l} c_{l}+\tilde{g}_{k-2 l} d_{l}\right)
$$

where $\left\{\tilde{h}_{k}, k \in \mathbb{Z}\right\}$ and $\left\{\tilde{g}_{k}, k \in \mathbb{Z}\right\}$ are the reconstruction lowpass and highpass filter coefficients, respectively, and they can be obtained from the following equations:

$$
g_{k}=(-1)^{k} \tilde{h}_{-k+1}, \quad \tilde{g}_{k}=(-1)^{k} h_{-k+1} .
$$

Based on the above fundamental backgrounds and some additional concepts, we will present several practical applications in the next section which will also indicate some advantages or disadvantages of different wavelet-based methods.

\section{A case study}

To examine statistics of signals and signal components is a very important task in studying the analysis of the well-known 1995 New Year's Day freak wave at the Draupner platform in the North Sea. In what follows, we will perform various wavelet analysis examinations on a given signal. We perform a multilevel wavelet decomposition, continuous wavelet transform with various wavelets, compression, denoising, as well as graphical examinational analysis of the corresponding wavelet coefficients which can be found in Figures 3.1, 3.2 3.3, 3.4, 3.5, 3.6, 3.7, 3.8, 3.9, 3.10, 3.11, 3.12, 3.13, 3.14, 3.15, 3.16, and 3.17. More precisely, we describe our studies of wavelet analysis for the given signal in more detail as follows. 

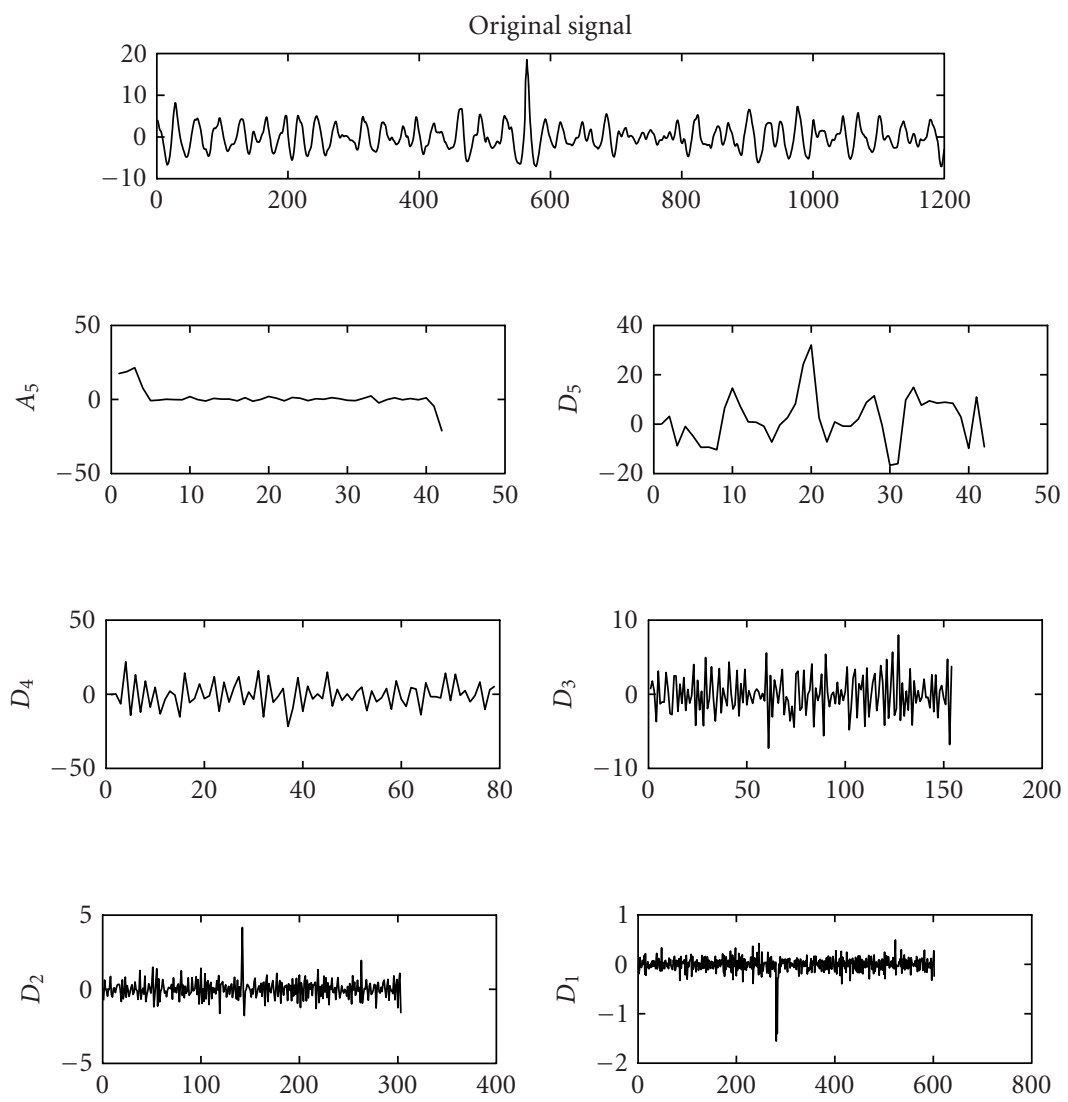

Figure 3.1. Decomposition of time series at level 5 with db3.

3.1. Multilevel decomposition. A multiresolution decomposition of the signal is performed in Figures 3.1 and 3.2. As indicated in (2.9) and (2.14) and shown in those figures as well, the higher level is more coarser, while the lower level is more finer and close to the original signal. The higher-level approximation shows a more smoother version of the signal, while the lower-level decomposition is less smoother and has similar smoothness to the original signal. Multilevel decomposition in the details indicates different natures of the signal. Wavelet decomposition produces a family of hierarchical decompositions. The selection of a suitable level for the hierarchy usually depends on the signal and experience. At each level $j$, the $j$-level approximation $A_{j}$ and a deviation signal called the $j$-level detail $D_{j}$ are the decompositions of the lower-level approximation $A_{j-1}$. For example, in Figure 3.1, the signal is decomposed as $A_{0}=A_{1}+D_{1}=A_{2}+D_{2}+D_{1}=\cdots=$ $A_{5}+D_{5}+D_{4}+D_{3}+D_{2}+D_{1}$. Perhaps it is of most interest that the freak wave effect, the one single unusually high wave, only appeared at low scales $D_{1}$ and $D_{2}$. So the freak wave is basically a time localized event not affected by higher scales or low-frequency wave processes. 
A discrete wavelet analysis of freak waves in the ocean
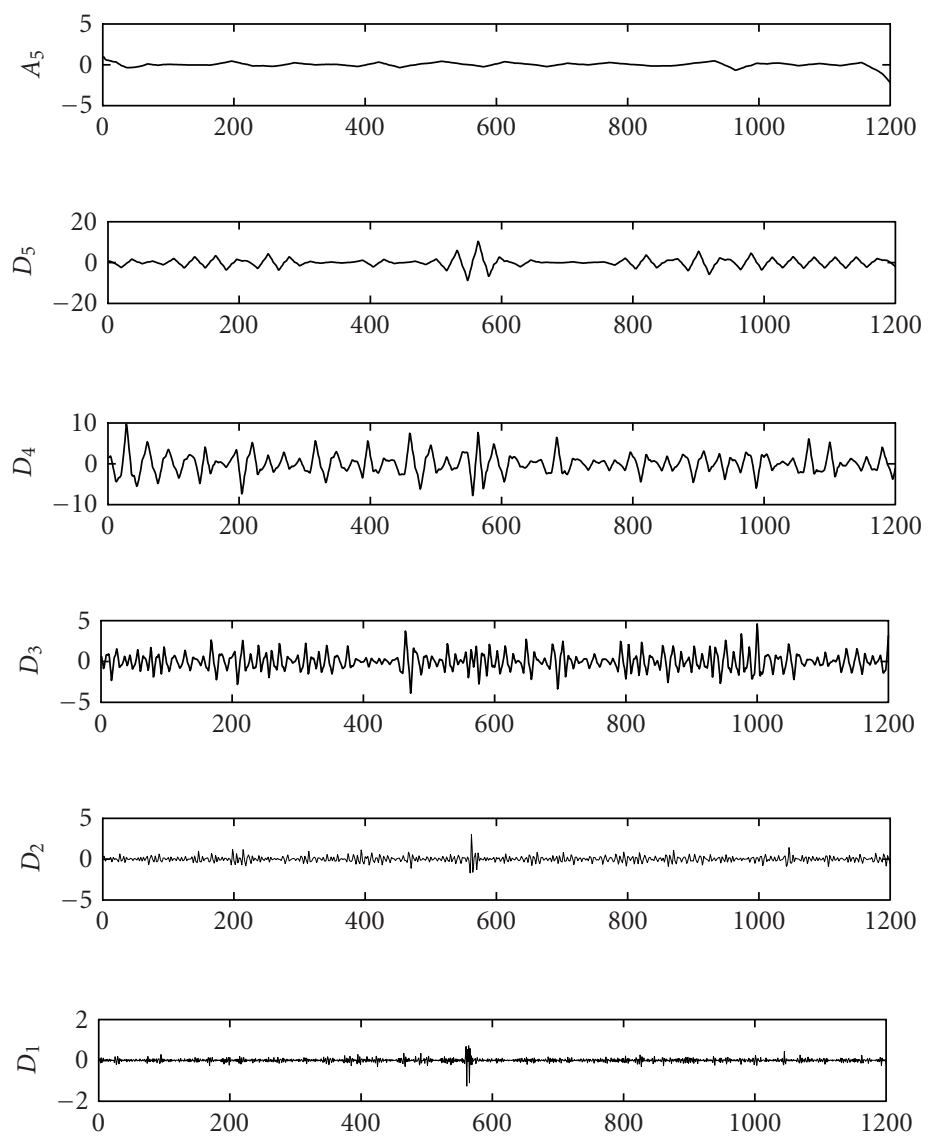

Figure 3.2. Approximation and details at level 5.

3.2. Compression. The compression of the signal is shown in Figure 3.4. Basically, the wavelet decomposition of the signal at level 5 is computed. For each level from 1 to 5 , a threshold (0.3490) is selected and hard thresholding is applied to the detail coefficients as described in Section 2. The residual and reconstruction from compression are also shown in the figure. Essentially the idea of compression is that some small detail components can be thrown out without appreciably changing the signal. Only the significant components need to be transmitted, and significant data compression can be achieved. The way to choose small detail components depends on the threshold chosen for a particular application.

3.3. Reconstruction. Computation results of wavelet reconstruction at level 5 with $\mathrm{db} 3$ and the modified detail coefficients of levels from 1 to 5 are shown in Figures 3.2 and 3.3. The corresponding reconstruction and approximation are plotted in those figures. A reconstruction algorithm was used so that the compressed signal can be rebuilt in terms of the basic elements generated by certain a scaling function (Figure 3.4). 


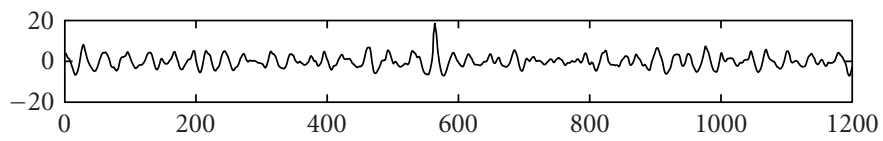

(a)

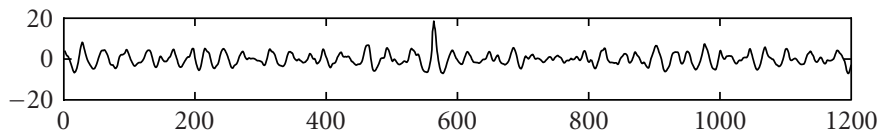

(b)

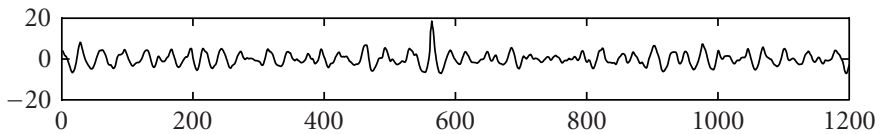

(c)

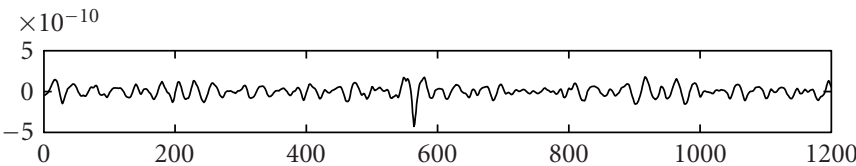

(d)

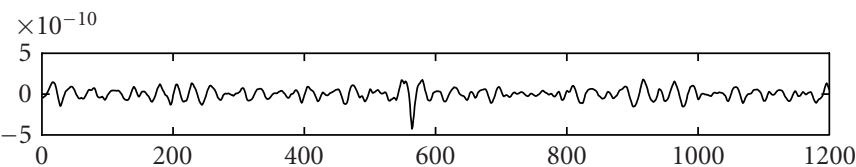

(e)

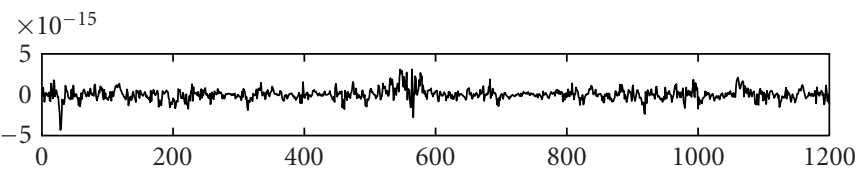

(f)

Figure 3.3. Reconstruction and approximation at level 5. (a) Original signal $X$. (b) $A_{0}$ : reconstructed from the multilevel wavelet decomposition. (c) Sum of approximation and details: $A_{5}+D_{5}+D_{4}+$ $D_{3}+D_{2}+D_{1}$. (d) $X-A_{0}$. (e) $X$-Sum. (f) $A_{0}$-Sum. 
386 A discrete wavelet analysis of freak waves in the ocean
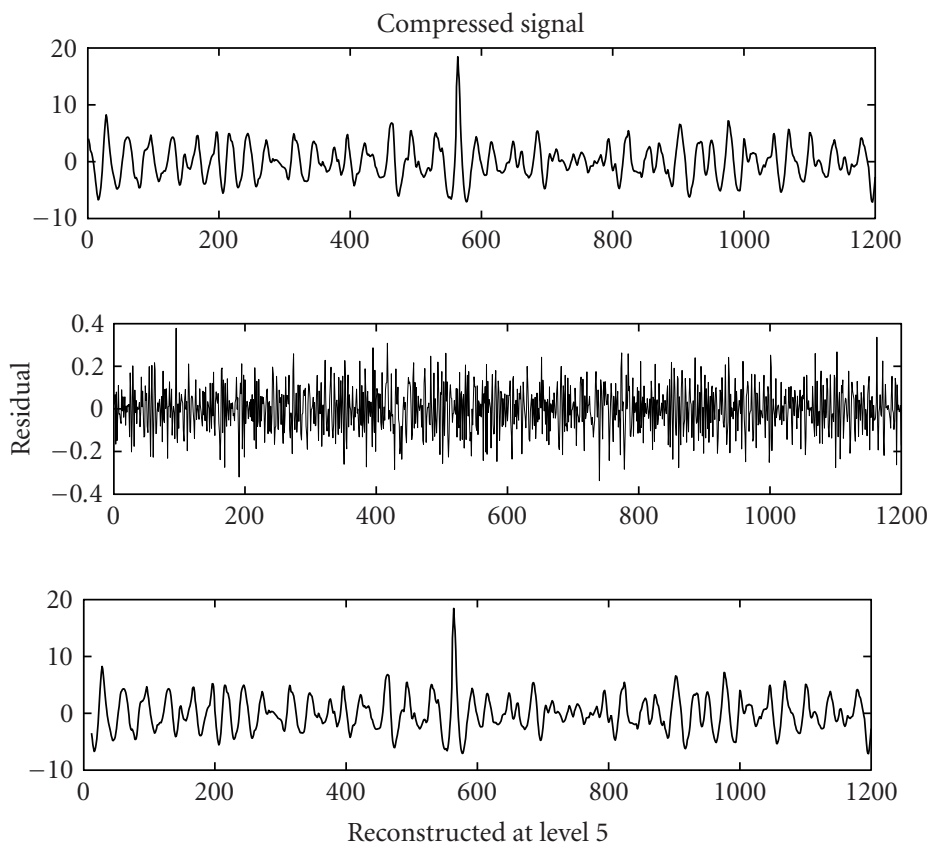

Figure 3.4. Compression with global thresholding.
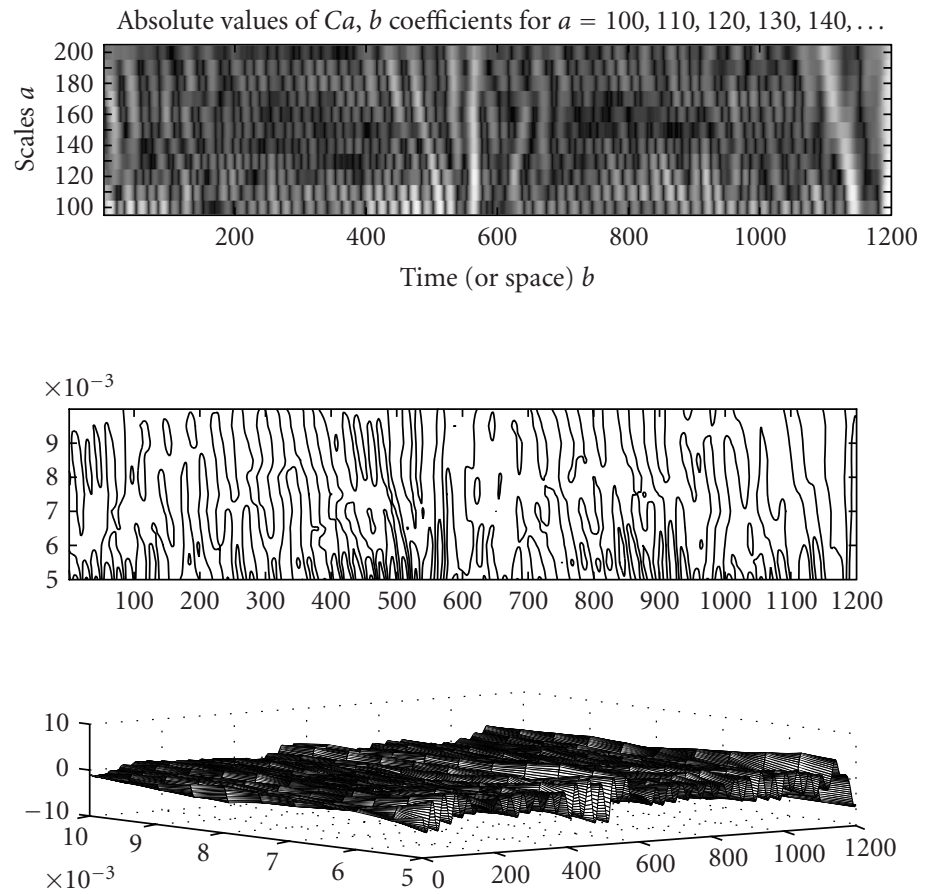

Figure 3.5. Continuous wavelet transform with $\mathrm{db} 3$. 
Absolute values of $C a, b$ coefficients for $a=100,110,120,130,140, \ldots$
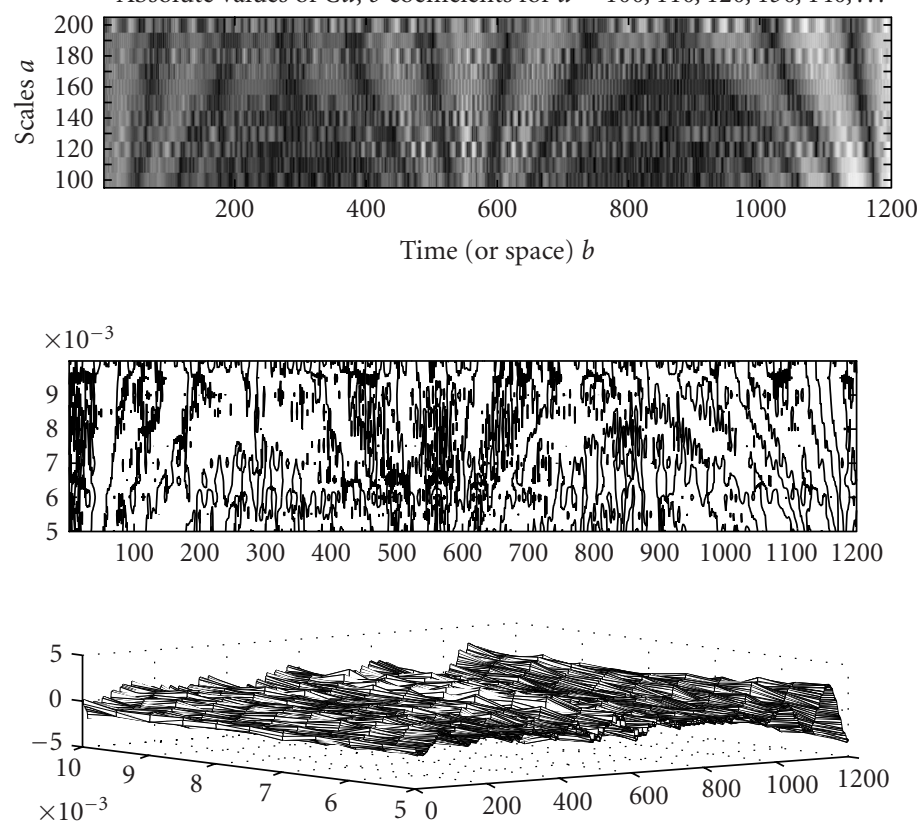

Figure 3.6. Continuous wavelet transform with morlet wavelet.
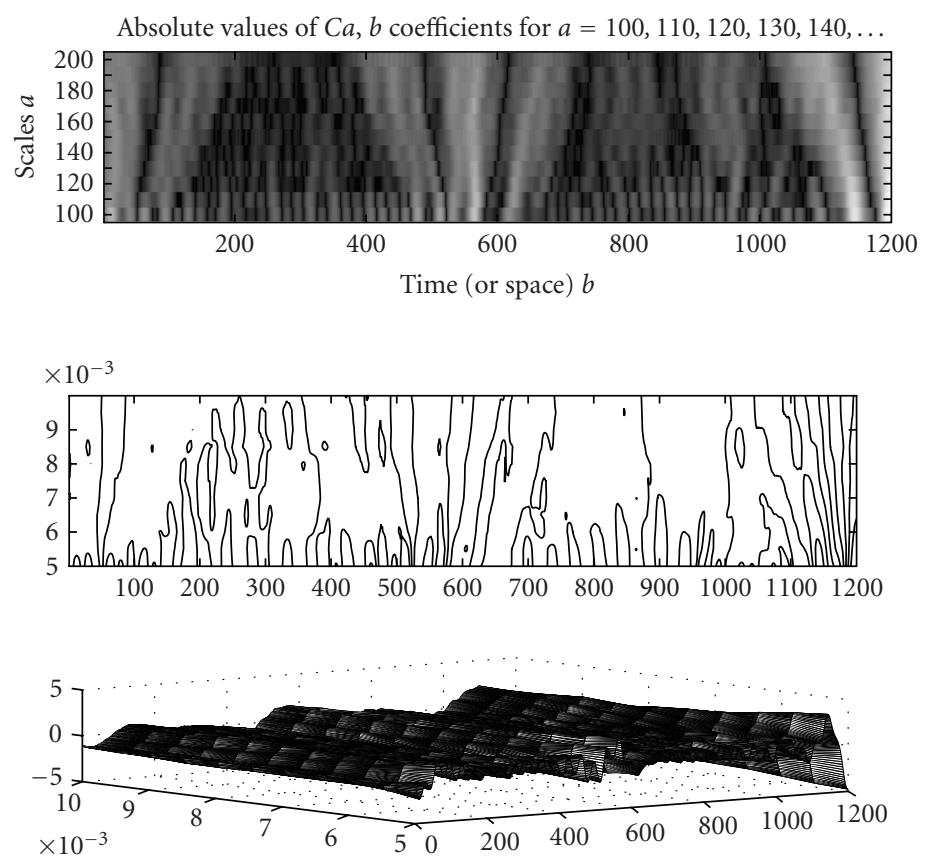

Figure 3.7. Continuous wavelet transform with Coiflet3. 
388 A discrete wavelet analysis of freak waves in the ocean
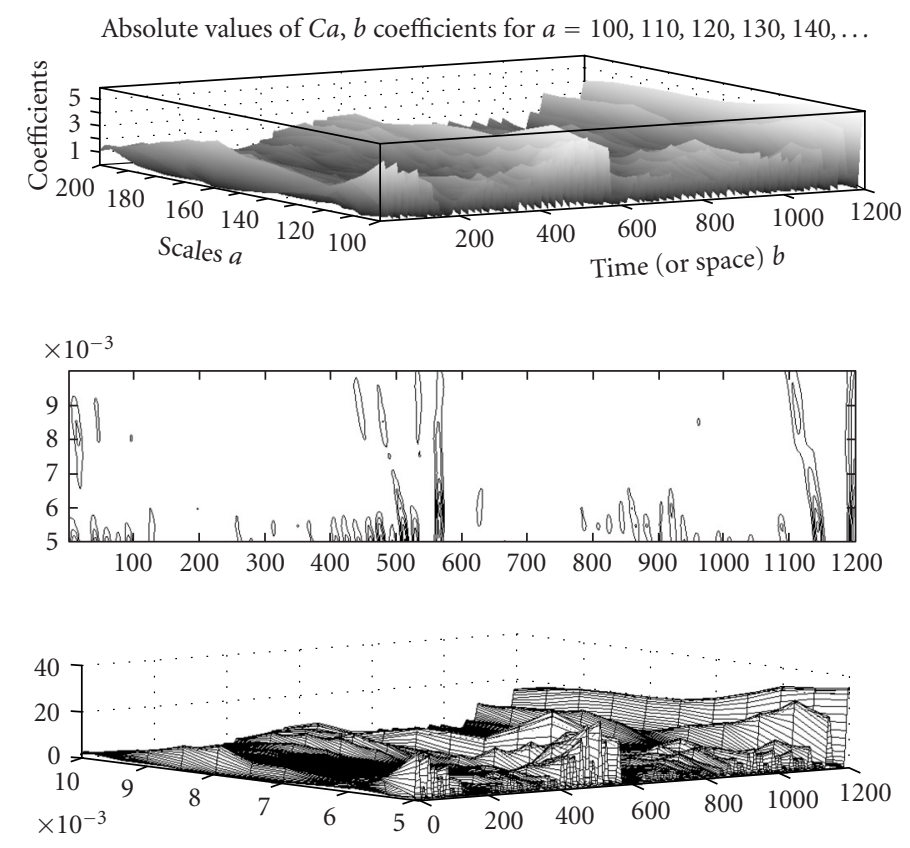

Figure 3.8. Wavelet spectrum with db3.
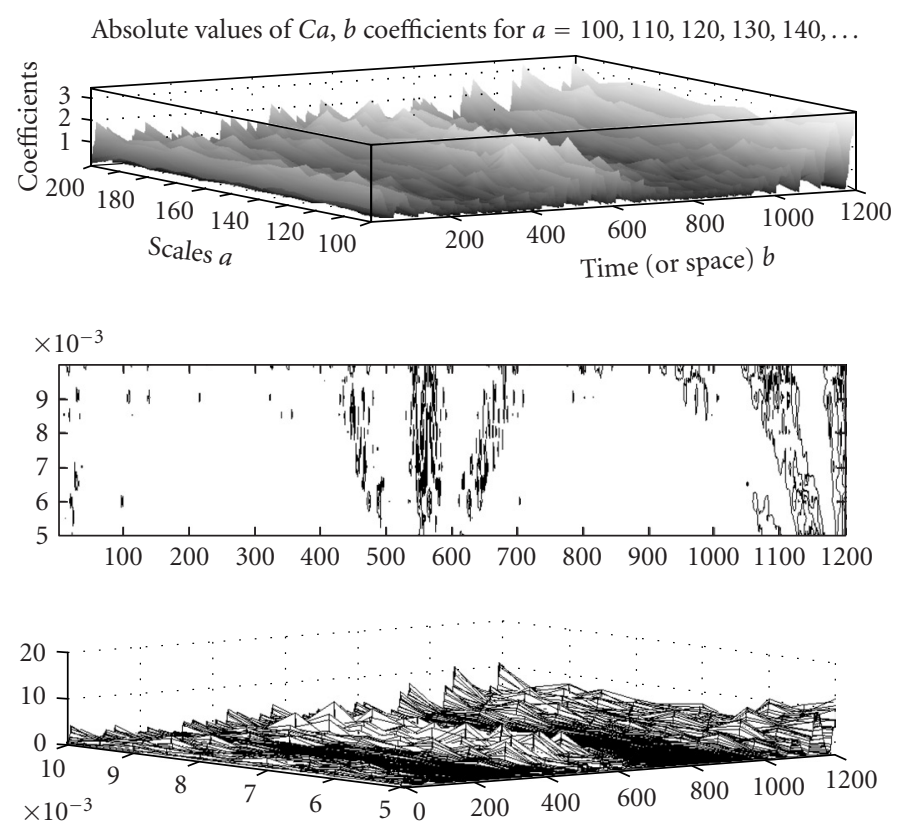

Figure 3.9. Wavelet spectrum with morlet. 
Absolute values of $C a, b$ coefficients for $a=100,110,120,130,140, \ldots$
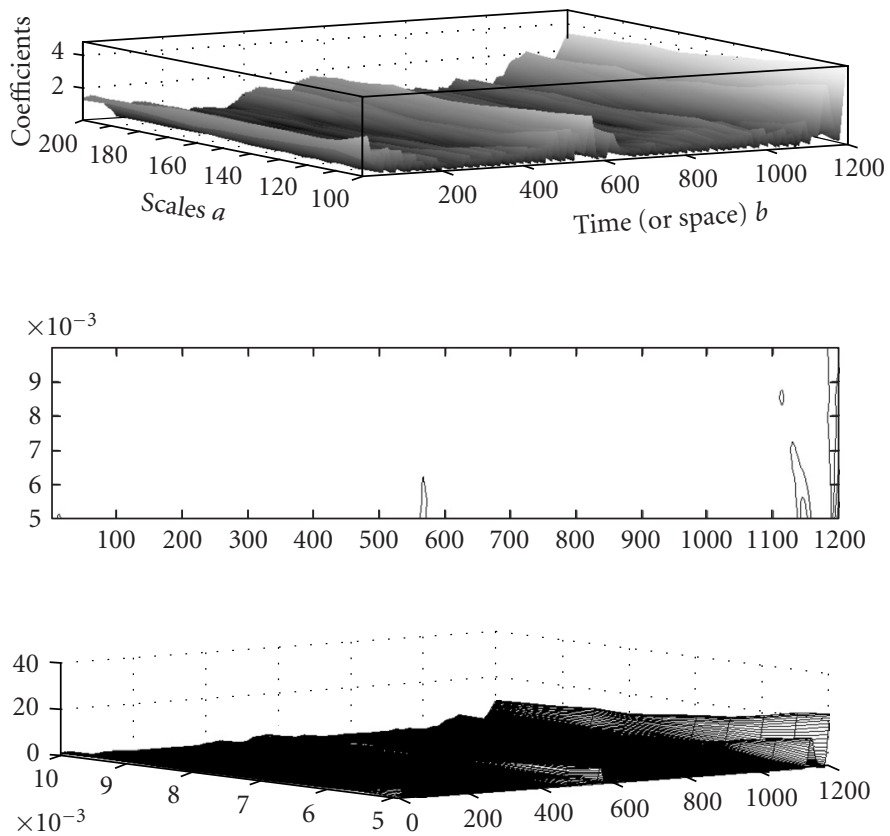

Figure 3.10. Wavelet spectrum with Coiflet3.
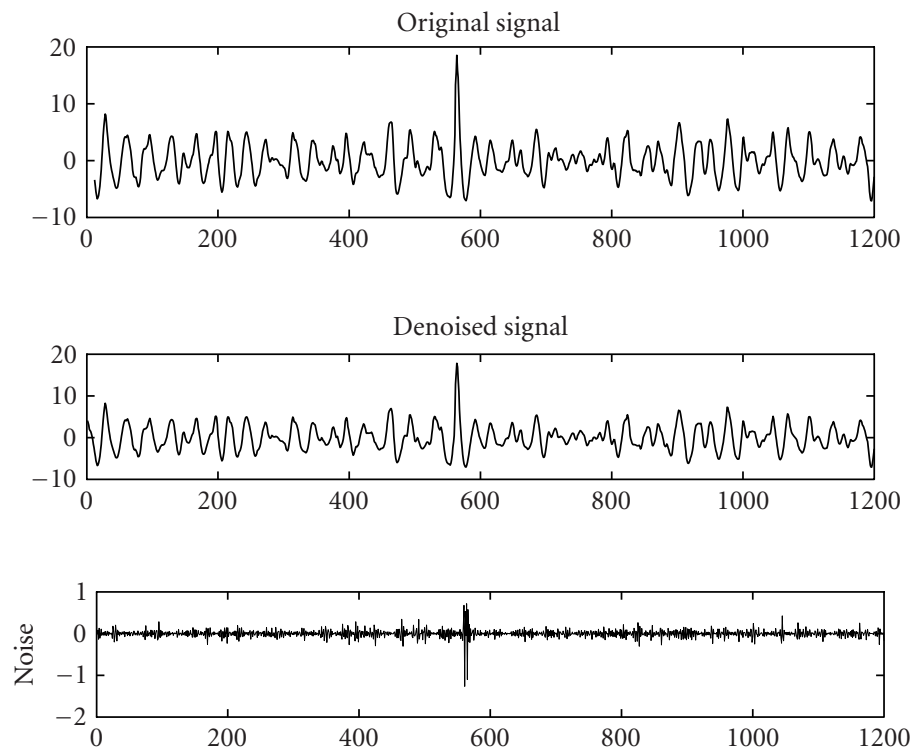

Figure 3.11. Denoising thr. db3 with global thresholding. (Zero Coefficients: 50\%, 2-norm reconstruction: $90.8868 \%$.) 
390 A discrete wavelet analysis of freak waves in the ocean
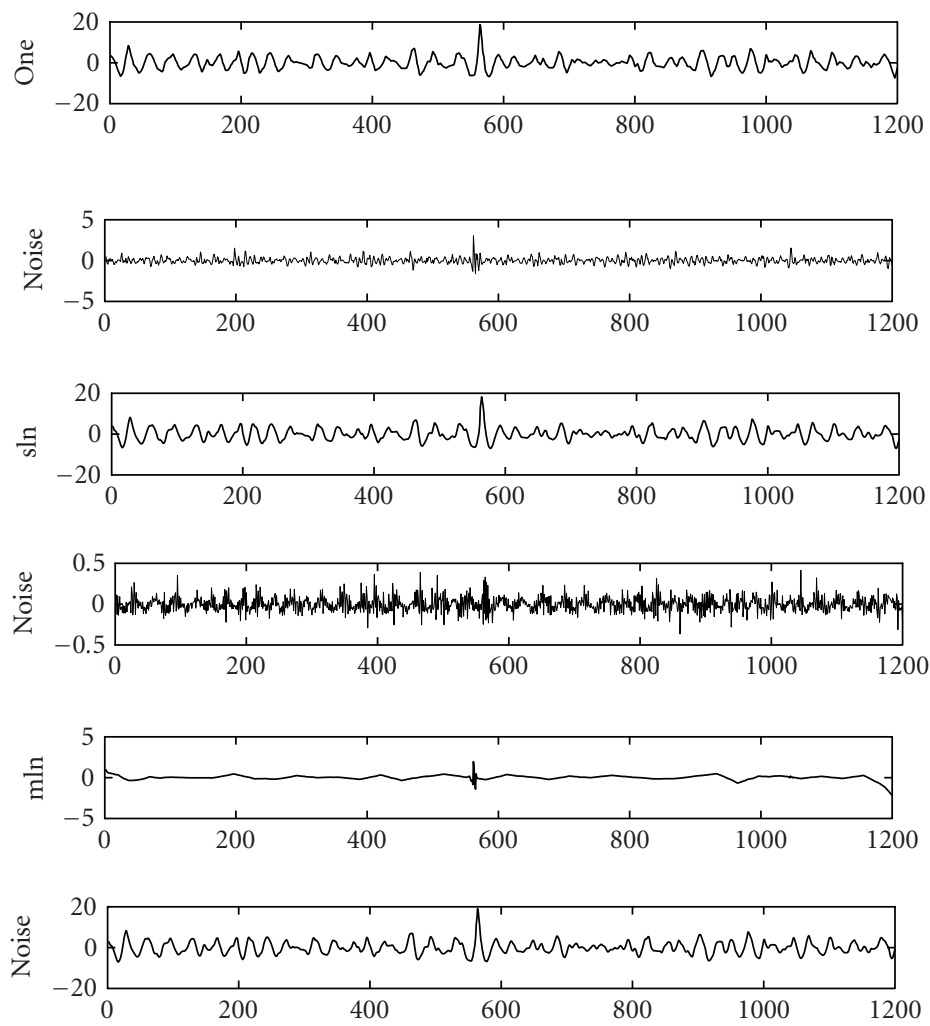

Figure 3.12. Denoised signal/basic /unscaled /nonwhite noise with db3.

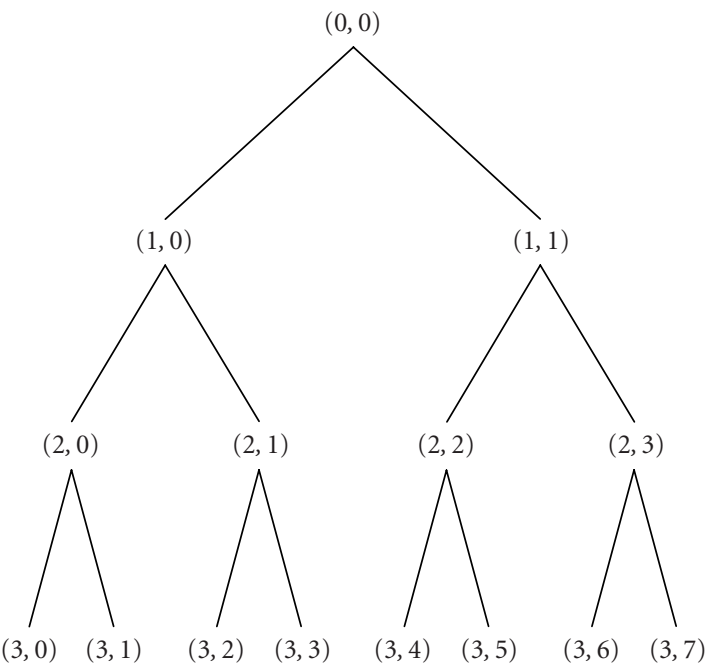

Figure 3.13. Best-level decomposition tree. 


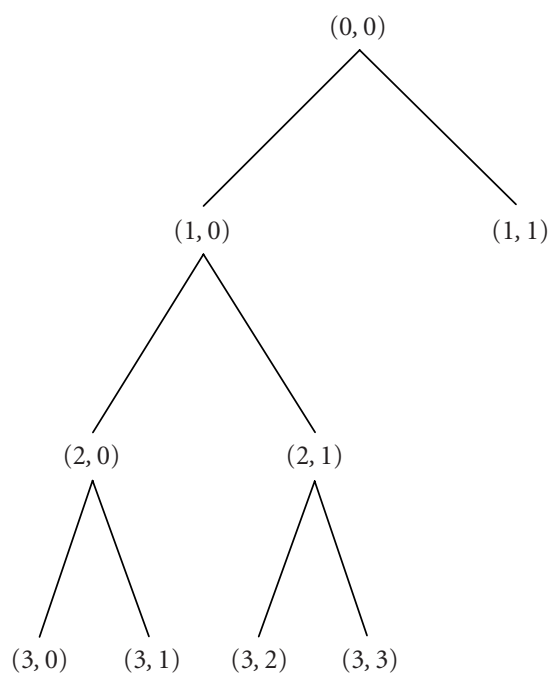

Figure 3.14. Wavelet packet with optimal subtree.
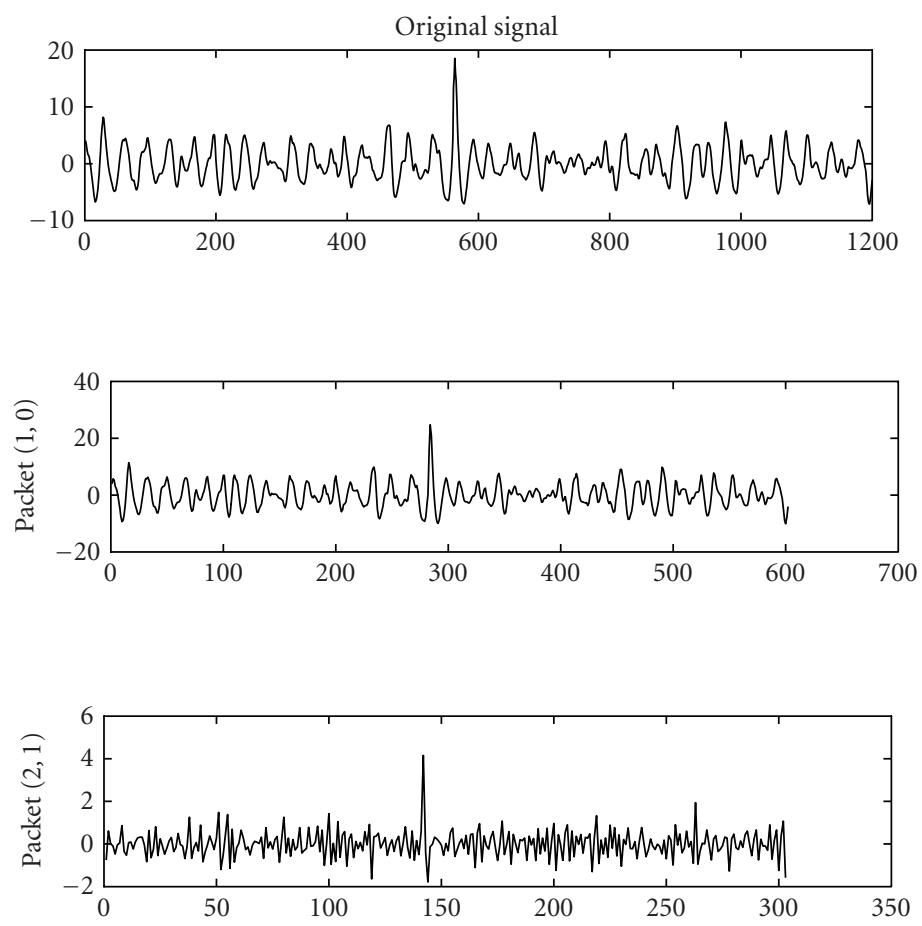

Figure 3.15. Wavelet packet with db3. 

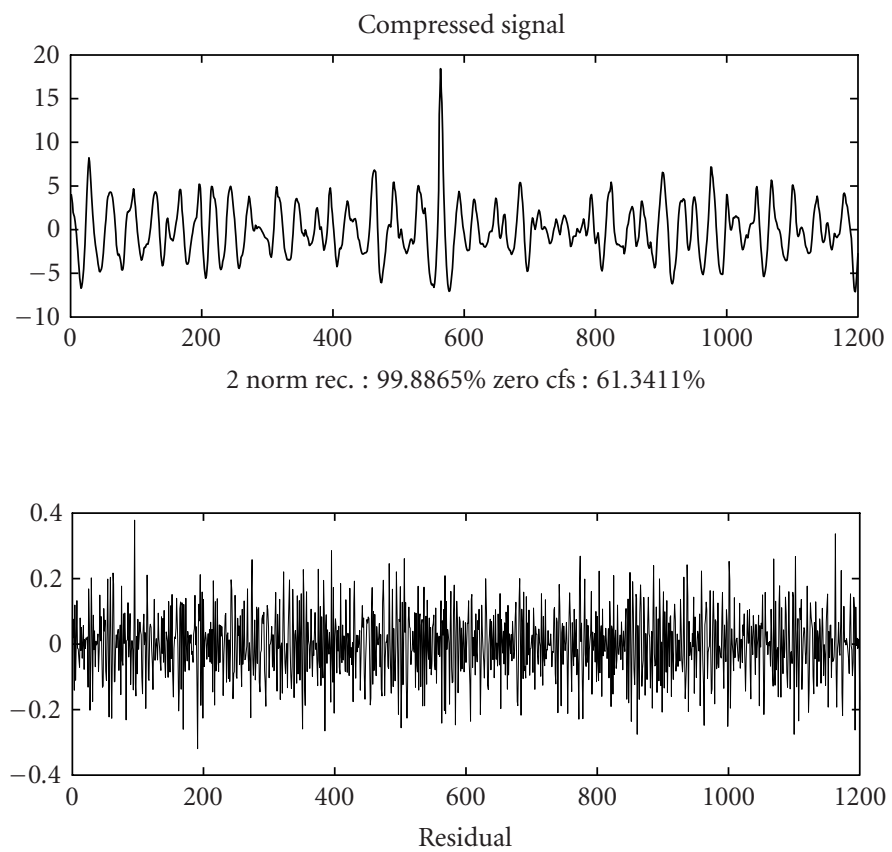

Figure 3.16. Compression thr. wavelet packet with $\mathrm{db} 3$.
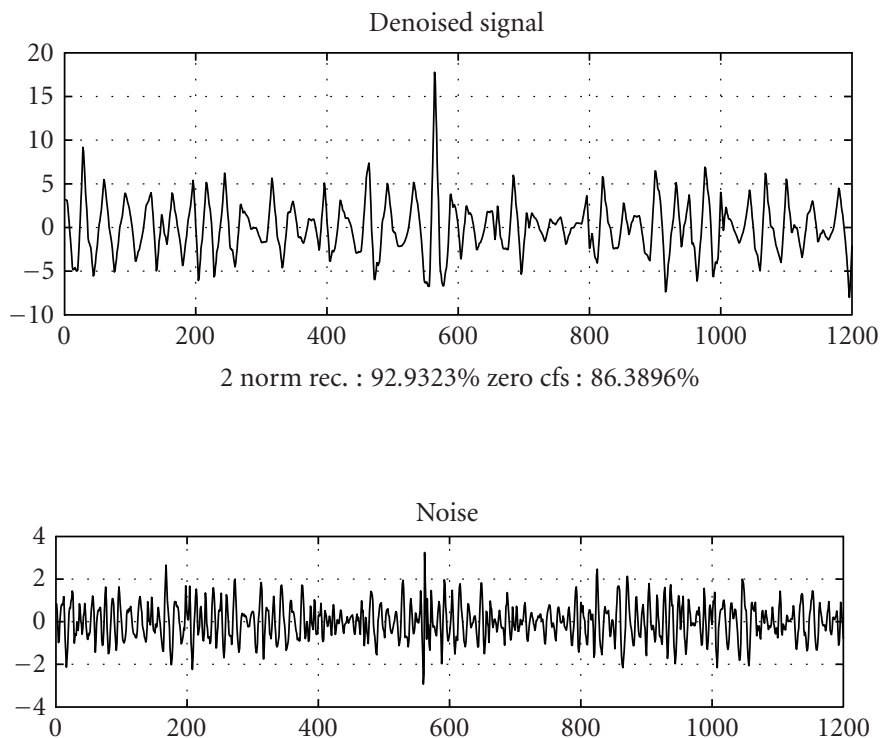

Figure 3.17. Denoising thr. wavelet packet with db3. 
3.4. Wavelet coefficients. Performing continuous wavelet transforms, we obtain several results. Wavelet coefficients of analyzed signals with $\mathrm{db} 3$ are shown in Figure 3.5. The corresponding spectrum is shown in Figure 3.8. Wavelet coefficients of analyzed signals with Morlet wavelet are shown in Figure 3.6. The corresponding spectrum is shown in Figure 3.9. Wavelet coefficients of analyzed signals with Coiflet 3 are shown in Figure 3.7. The corresponding spectrum is shown in Figure 3.10. The performances by using different wavelets can be viewed and compared while one searches for different natures and features of the corresponding wavelets. Again, the primary feature of freak waves is shown clearly at low scales in both discrete and continuous wavelet transform results. In Figures 3.5 to $3.10, C a, b$ is defined as (2.1).

3.5. Denoising. The denoising results based on wavelet decomposition with different noise assumptions are shown in Figures 3.11 and 3.12. The denoised signal in Figure 3.11 is obtained by wavelet coefficient thresholding using global threshold 1.9482. The threshold used in Figure 3.12 is a mixture of Stein's unbiased risk estimate and the square root of the signal length. The three denoising methods used are general basic model (one), basic model with unscaled noise $(\mathrm{sln})$, and basic model with nonwhite noise $(\mathrm{mln})$. As one can see from the resulting signals, comparing with the original wave, it turned out that the method with unscaled noise is a better denoising method.

3.6. Wavelet packet. The wavelet packet method is a generalization of wavelet decomposition as shown above. Wavelet packet atoms are waveforms indexed by three parameters: position, scale, and frequency. For a given signal with respect to a given orthogonal wavelet, we generate a library of bases called wavelet packet bases. Each of these bases offers a particular way of coding signals, preserving global energy, and reconstructing exact features. We obtain numerous expansions of a given signal by using wavelet packets. We then select the most suitable decomposition of a given signal with respect to an entropybased criterion. We analyze our signal into a decomposition tree with db3 as shown in Figure 3.13. We then select an optimal basis with the best level (Figure 3.14). The corresponding compression and denoising are shown in Figures 3.16 and 3.17, respectively. Some coefficients of subtrees are shown in Figure 3.15.

\section{Concluding remarks}

While this study is a first attempt in applying wavelet tools to exhaust various analyses on a set of freak wave data, it would be interesting to compare results for further studies on other waves or using different wavelets. It is also interesting to develop other studies by using interpolation methods [4] as well. Freak waves are a major threat to ships and offshore structures such as oil rigs, but they are difficult to predict. It seems that freak waves exist even in the ocean far away from strong current gradients. A wave must be at least 2.2 times the height of the largest $33 \%$ of the waves. Some freak waves are caused by strong currents or the chance reinforcement of two large waves, and a so-called "selffocusing" effect could also create outside waves [1]. The modeling of the waves from the point of view of the nonlinear Schrodinger equation was developed by several authors [1]. 
394 A discrete wavelet analysis of freak waves in the ocean

It would be interesting to construct a model and compare some simulations which will be compatible with the results derived from this paper.

\section{Acknowledgments}

The authors are grateful for the data courtesy of Sverre Haver of Statoil, Norway. The first author would like to thank Great Lakes Environmental Research Laboratory, NOAA, for their hospitality during his visit. This paper is GLERL contribution No. 1330.

\section{References}

[1] K. B. Dysthe and K. Trulsen, Note on breather type solutions of the NLS as model for freak waves, Phys. Scripta T82 (1999), 48-52.

[2] H. M. Fritz and P. C. Liu, An application of wavelet transform analysis to landslide-generated impulse waves, Ocean Wave Measurement and Analysis (San Francisco, 2000) (B. L. Edge and J. M. Hemsley, eds.), American Society of Civil Engineering, Virginia, 2001, pp. 14771486.

[3] P. A. E. M. Janssen, Nonlinear four-wave interactions and freak waves, J. Phys. Oceanogr. 33 (2003), no. 4, 863-884.

[4] E.-B. Lin, A survey on scaling function interpolation and approximation, Applied Mathematics Reviews, Vol. 1, World Scientific, New Jersey, 2000, pp. 559-607.

[5] P. C. Liu, Wavelet spectrum analysis and ocean wind waves, Wavelets in Geophysics (Maryland, 1993) (E. Foufoula-Georgiou and P. Kumar, eds.), Wavelet Anal. Appl., vol. 4, Academic Press, California, 1994, pp. 151-166.

[6] Wavelet transform and new perspective on coastal and ocean engineering data analysis, Advances in Coastal and Ocean Engineering Vol. 6 (P. Liu, ed.), World Scientific, 2000, pp. $57-101$.

[7] P. C. Liu and N. Mori, Characterizing freak waves with wavelet transform analysis, Rogue Waves 2000 (France) (M. Olagnon and G. A. Athanassoulis, eds.), Ifremer, France, 2001, pp. 151156.

[8] S. Mallat, A Wavelet Tour of Signal Processing, 2nd ed., Academic Press, California, 1999.

En-Bing Lin: Department of Mathematics, University of Toledo, Toledo, OH 43606, USA

E-mail address: elin@math.utoledo.edu

Paul C. Liu: Great Lakes Environmental Research Laboratory, National Oceanic and Atmospheric Administration (NOAA), Ann Arbor, MT 48105-2945, USA

E-mail address: paul.c.liu@noaa.gov 


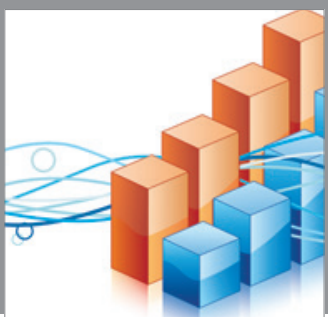

Advances in

Operations Research

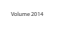

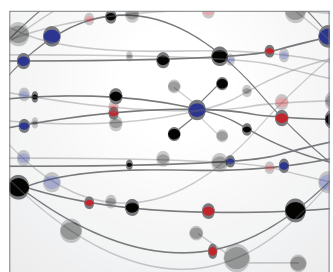

\section{The Scientific} World Journal
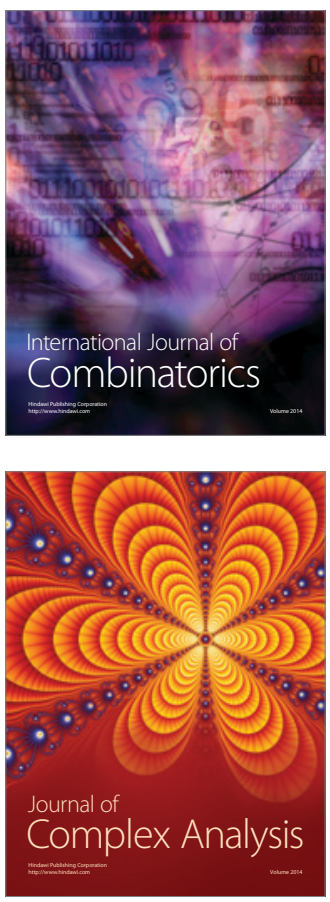

International Journal of

Mathematics and

Mathematical

Sciences
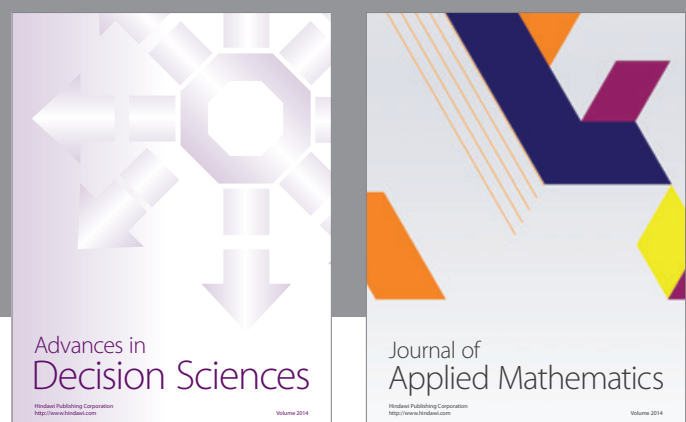

Journal of

Applied Mathematics
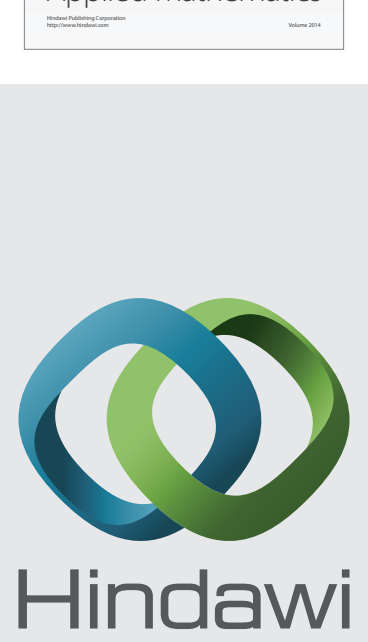

Submit your manuscripts at http://www.hindawi.com
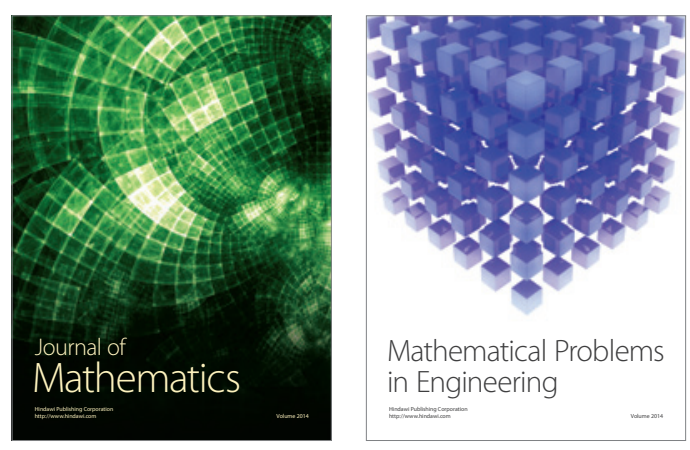

Mathematical Problems in Engineering
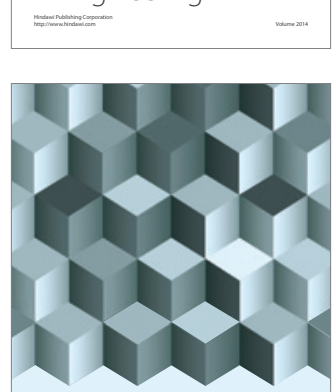

Journal of

Function Spaces
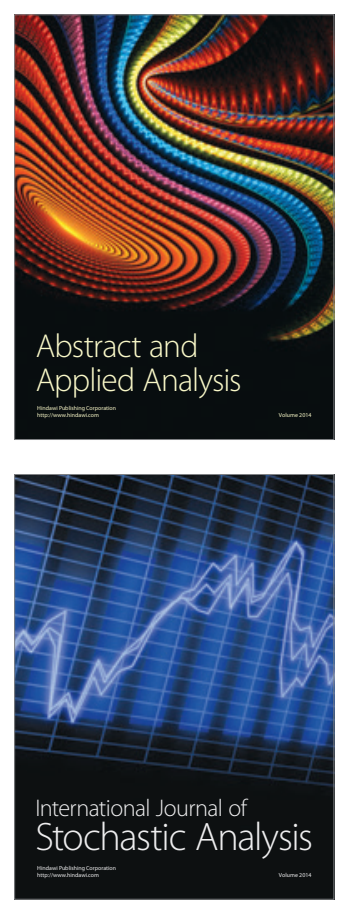

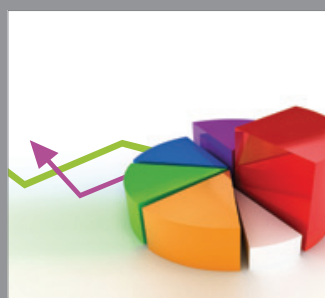

ournal of

Probability and Statistics

Promensencen
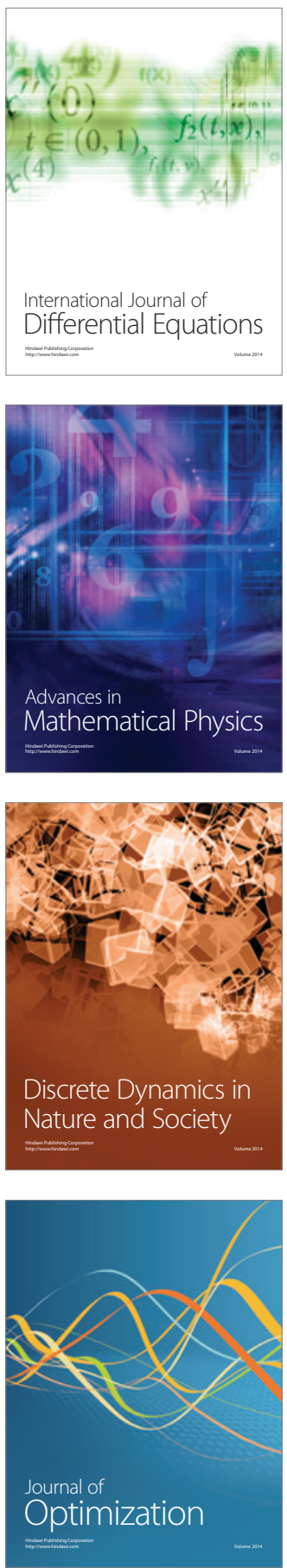